\title{
ENVELHECIMENTO ACELERADO EM DIÁSPOROS DE Oenocarpus bacaba (ARECACEAE) ${ }^{1}$
}

\author{
BRENO MARQUES DA SILVA E SILVA ${ }^{2}$, RUBENS SADER ${ }^{3}$, FABIOLA VITTI MORO $^{4}$, \\ CAMILA DE OLIVEIRA ${ }^{5}$, CÉSAR MARTORELI DA SILVEIRA ${ }^{6}$
}

RESUMO- O objetivo do presente trabalho foi adequar o teste de envelhecimento acelerado para avaliar a qualidade fisiológica de diásporos de Oenocarpus bacaba Mart. (bacaba). Inicialmente, os diásporos foram expostos às temperaturas de $41 ; 43$ e $45^{\circ} \mathrm{C}$, durante $3 ; 6 ; 12 ; 24 ; 36 ; 48$ e $72 \mathrm{~h}$, com cerca de $100 \%$ de umidade relativa do ar. Foram considerados dois critérios de germinação: emissão da primeira bainha igual ou superior a $1 \mathrm{~cm}$ e plântula normal. O teste de germinação foi realizado com 6 repetições de 20 diásporos, em temperatura de $30^{\circ} \mathrm{C}$ e fotoperíodo de $12 \mathrm{~h}$. Pelos resultados preliminares, observou-se que os lotes de diásporos de bacaba podem ser separados em níveis de vigor por meio do envelhecimento acelerado, em $43^{\circ} \mathrm{C}$ e com cerca de $100 \%$ de umidade relativa do ar, por $48 \mathrm{~h}$. Os quatro lotes de diásporos foram então submetidos, num segundo experimento, a $43^{\circ} \mathrm{C}$ por $24 ; 48$ e $72 \mathrm{~h}$, sendo adotada como critério de germinação a emissão de primeira bainha. Em $45^{\circ} \mathrm{C}$, a porcentagem de germinação foi drasticamente reduzida após $12 \mathrm{~h}$. Não houve diferenças significativas entre os critérios de germinação utilizados. Os lotes de diásporos foram separados em diferentes níveis de vigor após 48 e $72 \mathrm{~h}$ de exposição a $43{ }^{\circ} \mathrm{C}$. De acordo com os resultados obtidos, sugere-se o uso de primeira bainha como critério de germinação e período de envelhecimento de $48 \mathrm{~h}$ a $43^{\circ} \mathrm{C}$, para a avaliação da qualidade fisiológica de diásporos de bacaba.

Termos para indexação: vigor, germinação, viabilidade, Palmae.

\section{ACCELERATED AGING OF Oenocarpus bacaba (ARECACEAE) DIASPORES}

ABSTRACT - The evaluation of Oenocarpus bacaba diaspore physiological quality by means of the accelerated aging test was studied. The diaspores were submitted to temperatures of 41,43 , and $45^{\circ} \mathrm{C}$ for $3,6,12,24,36,48$, and $72 \mathrm{~h}$ at a relative humidity of $100 \%$. Two germination criteria were employed to evaluate the results: 1 . emission of the first $1 \mathrm{~cm}$ or longer sheath, and 2 . normal seedling. The germination test was carried out with 6 replications of 20 diaspores at a temperature of $30^{\circ} \mathrm{C}$ and a $12 \mathrm{~h}$ photoperiod. Based on preliminary results, four diaspore lots were submitted to $43{ }^{\circ} \mathrm{C}$ for 24,48 , and $72 \mathrm{~h}$ and the evaluation of germination was based on the emission of the first sheath. At $45^{\circ} \mathrm{C}$, germination percentage was severely reduced after $12 \mathrm{~h}$. The use of the first sheath is suggested as germination criterion. The diaspore lots were more clearly separated when the accelerated aging test was conducted for $48 \mathrm{~h}$ at the temperature of $43{ }^{\circ} \mathrm{C}$.

Index terms: germination, vigor, viability, Palmae.

\section{INTRODUÇÃO}

Oenocarpus bacaba Mart. é uma palmeira nativa do Brasil, utilizada em construções rústicas, paisagismo e de cujos frutos é produzido vinho, muito consumido pela população da região Amazônica. A qualidade fisiológica das sementes normalmente é avaliada pelos testes de germinação e de vigor. Entre os testes de vigor, o de envelhecimento acelerado tem sido estudado para diferentes espécies, com o intuito de definir as condições de temperatura e tempo de exposição das sementes, sob alta umidade relativa $(100 \%)$, baseando-se no fato de que a taxa de deterioração das sementes aumenta consideravelmente quando submetidas a tais condições (VIEIRA \& CARVALHO, 1994; MARCOS FILHO, 1999; 2005).

No envelhecimento acelerado, o processo de deterioração é semelhante ao do envelhecimento

\footnotetext{
${ }^{1}$ (Trabalho 099-08). Recebido em: 18-04-2008. Aceito para publicação em: 08-10-2009.Parte da Dissertação de Mestrado em Agronomia [Produção e Tecnologia de Sementes] - UNESP Jaboticabal - 2007.

${ }^{2}$ CAPES - Doutorando Agronomia [Produção e Tecnologia de Sementes] - Depto. de Produção Vegetal (DPV) - FCAV - UNESP Jaboticabal. E-mail: silvabms@hotmail.com

${ }^{3}$ Prof. Titular Dr. - UNESP Jaboticabal - DPV - FCAV - UNESP Jaboticabal. E-mail: rsader@fcav.unesp.br

${ }^{4} \mathrm{CNPq}$ - Nível 2 - Prof. Assistente Dra. - Depto. de Biologia Aplicada à Agropecuária (DBAA) - FCAV - UNESP Jaboticabal. Email: fabiola@fcav.unesp.br

${ }^{5}$ Bacharelanda em Ciências Biológicas - Universidade de Franca (UNIFRAN). E-mail: kamilaoliveira@gmail.com

${ }^{6}$ CAPES - Doutorando em Agronomia [Produção Vegetal] - DPV - FCAV - UNESP Jaboticabal. E-mail: cmspirangi@hotmail.com
} 
natural das sementes, porém a uma velocidade acelerada (DELOUCHE \& BASKIN, 1973; TEKRONY, 1993; VIEIRA \& CARVALHO, 1994). As sementes mais vigorosas deterioram-se mais lentamente que as menos vigorosas, apresentando redução diferenciada da viabilidade (TORRES, 2004). De acordo com TOMES et al. (1988), a elevação da temperatura promove efeitos mais drásticos que o prolongamento do período de exposição ao envelhecimento artificial.

Nesse contexto, o uso de testes que forneçam uma estimativa do desempenho das sementes em campo e/ou armazenamento é um aspecto importante a ser considerado em um programa de produção de sementes. Estes testes, que avaliam o vigor das sementes, são indicados para identificar diferenças entre lotes, principalmente daqueles que possuem porcentagem de germinação semelhante (VIEIRA \& CARVALHO, 1994; MARCOS-FILHO, 1999).

De acordo com NEGREIROS \& PEREZ (2004), as sementes de Dypsis lutescens (H. Wendl.) Beentje \& J. Dransf. e Euterpe edulis Mart. são sensíveis ao envelhecimento acelerado, e as sementes de Phoenix reclinata Jacq. mantêm-se viáveis até $72 \mathrm{~h}$ após envelhecimento acelerado a $45^{\circ} \mathrm{C}$. MURUGESAN et al. (2005) relataram que o envelhecimento acelerado em $40^{\circ} \mathrm{C}$, por 70 dias, proporciona a aceleração e uniformização da germinação de sementes de Elaeis guianensis Jacq. var. dura Becc.

Em virtude da falta de estudos sobre a germinação e o vigor e, igualmente, de previsão para o armazenamento de diásporos, o objetivo do presente trabalho foi adequar o teste de envelhecimento acelerado para avaliar a qualidade fisiológica de diásporos de Oenocarpus bacaba Mart.

\section{MATERIAL E MÉTODOS}

Coleta e formação dos lotes: em semanas consecutivas, no mês de março de 2005, os frutos de bacaba para formar os lotes A, B, C e D foram coletados de 10 matrizes localizadas na Área de Proteção Ambiental do Rio Curiaú, Macapá-AP, Brasil. Na primeira semana, foi coletado o lote D e, nas semanas subsequentes, os lotes B, C e A. Os lotes coletados inicialmente foram mantidos em sacos de papel e em temperatura ambiente até a colheita do último lote (lote A). Os lotes foram enviados para as avaliações no Laboratório de Análise de Sementes, Departamento de Produção Vegetal, Faculdade de Ciências Agrárias e Veterinárias/FCAV, Universidade Estadual Paulista/UNESP, Jaboticabal-SP, Brasil.

Determinação do teor de água e qualidade fisiológica dos lotes: os frutos foram imersos em água morna $\left(47 \pm 3^{\circ} \mathrm{C}\right)$ durante $15 \mathrm{~min}$ e, poste- riormente, o epicarpo e o mesocarpo foram retirados por meio de fricção manual contra peneira de malha de aço. O teor de água dos diásporos dos lotes $\mathrm{A}, \mathrm{B}$, $\mathrm{C}$ e $\mathrm{D}$ foi determinado com quatro repetições de 10 diásporos em estufa a $105^{\circ} \mathrm{C} \pm 3$, durante $24 \mathrm{~h}$.

Para avaliar a qualidade fisiológica dos lotes A, B, C e D, seis repetições de 20 diásporos foram colocadas para germinar em caixas plásticas, sobre areia lavada e esterilizada, umedecida com solução aquosa de Benlate a $0,1 \%$, mantidas em germinador em $30^{\circ} \mathrm{C}$ e fotoperíodo de $12 \mathrm{~h}$, de acordo com indicações de SILVA et al. (2005). Decorridos 90 dias de semeadura, foram determinadas a altura das plântulas (do ápice radicular até o ápice do folíolo) e a espessura do colo (CLEMENT \& BOVI, 2000), assim como a massa de matéria seca das plântulas, por meio da secagem em estufa a $70^{\circ} \mathrm{C}$, durante 72 h (BENINCASA, 2003).

Experimento 1: Padronização da temperatura e do período de envelhecimento acelerado: para se determinar a temperatura e o período de envelhecimento adequados, os diásporos do lote $\mathrm{A}$ foram colocados sobre uma tela de aço, em caixas plásticas, contendo $40 \mathrm{~mL}$ de água destilada e deionizada (MARCOS FILHO, 1999), mantidas em germinadores a $41 ; 43$ e $45^{\circ} \mathrm{C}$, por $3 ; 6 ; 12 ; 24 ; 36$; 48 e 72 h. Em seguida, procedeu-se a germinação com seis repetições de 20 diásporos, segundo SILVA et al. (2005). Para a avaliação da germinação, foram usados dois critérios: o desenvolvimento de pelo menos $1 \mathrm{~cm}$ da primeira bainha e a formação de plântula normal, de acordo com SILVA (2007).

Experimento 2: Avaliação do vigor dos lotes pelo envelhecimento acelerado: os quatro lotes de diásporos foram colocados sobre tela de aço, em caixas plásticas contendo $40 \mathrm{~mL}$ de água destilada e deionizada, em germinador a $43^{\circ} \mathrm{C}$, por $24 ; 48$ e $72 \mathrm{~h}$. Posteriormente, procedeu-se a germinação conforme SILVA et al. (2005), porém apenas considerou-se como critério germinativo o desenvolvimento de pelo menos $1 \mathrm{~cm}$ de primeira bainha. $\mathrm{O}$ teor de água dos diásporos foi determinado para os lotes de diásporos, temperaturas e períodos de envelhecimento acelerado (BRASIL, 1992).

Foram calculados as porcentagens e os tempos médios de germinação dos diásporos, de acordo com LABOURIAU \& AGUDO (1987), utilizando-se da expressão $\mathrm{t}=\sum \mathrm{n}_{\mathrm{i}} \mathrm{t} / \sum \mathrm{n}_{\mathrm{i}}$, onde $\mathrm{t}=$ tempo médio de germinação; $n_{i=}$ número de sementes que germinaram no tempo $t_{i} ; t_{i=}$ tempo entre o início do experimento e a iésima observação (dia ou hora).

Foi utilizado o delineamento experimental inteiramente casualizado, sendo que, para o primeiro experimento, nas parcelas, foi utilizado o esquema 
fatorial $2 \times 3$, com dois critérios de germinação $(\geq 1 \mathrm{~cm}$ de primeira bainha e plântula normal) e três temperaturas de exposição $\left(41 ; 43\right.$ e $\left.45^{\circ} \mathrm{C}\right)$ e, nas subparcelas, sete períodos de envelhecimento acelerado $(3 ; 6 ; 12$; 24; 36; 48 e 72 h). Para o segundo experimento, nas parcelas, quatro lotes (A, B, C e D) e, nas subparcelas, três períodos de envelhecimento. Foi utilizado o teste $\mathrm{F}$, e, quando houve significância, as comparações entre as médias foram realizadas mediante a aplicação do teste de Tukey, a 5\% de probabilidade (PIMENTEL-GOMES, 1987).

\section{RESULTADOS E DISCUSSÃO}

Os teores de água iniciais dos lotes A, B, C e D foram altos e diferentes entre si (Tabela 1). MARTINS et al. (1999) e NEGREIROS \& PEREZ (2004) observaram valores de $35 ; 37$ e $51 \%$ para o teor de água de lotes de sementes de Dypsis lutescens, Euterpe edulis e Euterpe espiritosantensis (Fernandes), respectivamente.

Experimento 1: Padronização da temperatura e do período de envelhecimento acelerado: antes do envelhecimento, os lotes A e B apresentaram maior porcentagem de germinação, em relação aos lotes C e D. Pelo tempo médio de germinação, espessura do colo e massa seca das plântulas, não foi possível diferenciar os lotes A, B e C (Tabela 1).

Dentre os parâmetros testados inicialmente, o comprimento de plântulas foi o menos eficiente para diferenciar os lotes (Tabela 1). Ao contrário, essa característica foi eficiente para separar lotes de sementes de palmito-vermelho em diferentes níveis de qualidade fisiológica (MARTINS et al., 1999).

A absorção de água pelos diásporos do lote A não foi elevada, mesmo quando expostos ao envelhecimento acelerado a $41 ; 43$ e $45^{\circ} \mathrm{C}$ pelo período de $72 \mathrm{~h}$, provavelmente, devido ao alto teor de água inicial, em torno de $36 \%$.

A $41^{\circ} \mathrm{C}$, a porcentagem de germinação manteve-se constante, mesmo após $72 \mathrm{~h}$ de envelhecimento acelerado. Em contraste, a $43^{\circ} \mathrm{C}$, a porcentagem de germinação declinou a partir de $36 \mathrm{~h}$, havendo redução abrupta a $45^{\circ} \mathrm{C}$, quando comparado com 41 e $43^{\circ} \mathrm{C}$ (Figura 1). De acordo com NEGREIROS \& PEREZ (2004), a germinação de sementes de Euterpe edulis e Dypsis lutescens é suprimida totalmente em $45^{\circ} \mathrm{C}$ a partir de $12 \mathrm{~h}$ de envelhecimento acelerado.

Após $72 \mathrm{~h}$ de envelhecimento acelerado a $45^{\circ} \mathrm{C}$, a germinação dos diásporos de bacaba foi quase totalmente suprimida (Figura 1). O mesmo foi observado por NEGREIROS \& PEREZ (2004), com sementes de Phoenix reclinata e de Roystonea oleracea (Jacq.) O.F. Cook.).

Para o tempo médio de germinação, houve interação significativa entre o critério de avaliação de germinação, temperatura e os períodos de envelhecimento acelerado para os diásporos de bacaba. Desta forma, há diferenças básicas entre os critérios de germinação, pois os mesmos são separados naturalmente pelo tempo. Já a interação entre as temperaturas e os períodos de envelhecimento acelerado, novamente, indicaram haver diferenças para o aumento no tempo médio de germinação em função dos períodos de envelhecimento (Figura 2).

Em $72 \mathrm{~h}$ de envelhecimento acelerado, os diásporos de bacaba, independentemente da temperatura de exposição, apresentaram diminuição do tempo médio de germinação para o critério plântula normal. Ao contrário, NEGREIROS \& PEREZ (2004) relataram que as espécies Dypsis lutescens e Euterpe edulis apresentam maior sensibilidade aos efeitos adversos do envelhecimento acelerado, enquanto o período de $48 \mathrm{~h}$ de envelhecimento acelerado é crítico para vários parâmetros de vigor avaliados nas espécies Phoenix reclinata e Roystonea oleracea. De acordo com MURUGESAN et al. (2005), a germinação de sementes de dendê é acelerada depois de 70 dias de exposição ao envelhecimento acelerado a $40^{\circ} \mathrm{C}$.

Independentemente do critério de germinação, os tempos médios de germinação foram constantes em 41 e $43^{\circ} \mathrm{C}$ durante os períodos de envelhecimento acelerado para os diásporos de bacaba. No entanto, em $45^{\circ} \mathrm{C}$, houve uma redução do tempo médio de germinação quando o envelhecimento foi por um período de $72 \mathrm{~h}$ (Figura 2). Provavelmente, o aumento na velocidade de germinação dos diásporos de bacaba em $45^{\circ} \mathrm{C}$, por $72 \mathrm{~h}$ de envelhecimento, esteja relacionado com a germinação de poucos diásporos (Figura 1), porém de alto vigor dentro do lote A, pois sementes de alto vigor geralmente germinam mais rapidamente (VIEIRA \& CARVALHO, 1994; CARVALHO \& NAKAGAWA, 2000).

Experimento 2: Avaliação do vigor dos lotes pelo envelhecimento acelerado: Para a comparação dos lotes de diásporos durante o envelhecimento acelerado, foram adotados como critérios: a emissão da primeira bainha, a temperatura de $43^{\circ} \mathrm{C}$ e períodos de envelhecimento de $24 ; 48$ e $72 \mathrm{~h}$, pois não houve diferença significativa entre os critérios primeira bainha e plântula normal, assim como a temperatura de $41^{\circ} \mathrm{C}$ não foi eficiente na redução da capacidade germinativa, e, em $45^{\circ} \mathrm{C}$, a diminuição da germinação foi abrupta (Figura 1).

Os lotes A e B não absorveram muita água durante o envelhecimento acelerado quando compa- 
rados com os lotes $\mathrm{C}$ e $\mathrm{D}$, com valores de, respectivamente, 36,$3 ; 35,9 ; 35,1$ e 33,6 . Provavelmente, a baixa absorção de água pelos diásporos dos lotes está associada ao alto conteúdo de água inicial destes lotes (Tabela 1).

Depois de $24 \mathrm{~h}$ de envelhecimento acelerado a $43^{\circ} \mathrm{C}$, para a porcentagem de germinação, não houve diferenciação entre os lotes A e B (Figura 3). Desta forma, os resultados foram semelhantes aos observados inicialmente (Tabela 1). No entanto, a partir de $48 \mathrm{~h}$ e $72 \mathrm{~h}$, foi possível separar os lotes A e B, pois os mesmos tiveram germinação diferente (Tabela 1 e Figura 3). Como após 48 h de envelhecimento acelerado foi possível separar os lotes em diferentes níveis de vigor, esse período foi adotado como adequado.

Independentemente dos lotes, não houve diferenças significativas para os tempos médios de germinação dos diásporos entre os períodos de envelhecimento acelerado. Já para os lotes, independentemente do período de exposição, a germinação do lote D foi mais lenta em relação aos demais (24,9 dias). Isso encontra-se de acordo com a literatura citada, onde, em geral, os lotes de sementes de menor vigor apresentam germinação mais lenta (CARVALHO \& NAKAGAWA, 2000).

TABELA 1 - Teor de água inicial (TA); porcentagem de germinação considerando-se como critério a emissão da primeira bainha (PB) e plântula normal (PN); tempo médio de germinação considerando-se como critério a primeira bainha (TMB) e plântula normal (TMPN); comprimento de plântulas (CP); espessura do colo (EC) e massa seca (MS) de plântulas de Oenocarpus bacaba Mart., antes do envelhecimento acelerado.

\begin{tabular}{cllllllll}
\hline Lote & TA(\%) & $\mathbf{P B}(\%)$ & TMB(Dia) & $\mathbf{P N}(\%)$ & TMPN(Dia) & $\mathbf{C P ( c m )}$ & $\mathbf{E C ~ ( m m ) ~}$ & MS (g) \\
\hline $\mathbf{A}$ & 35,4 & $94,2 \mathrm{a}$ & $21,9 \mathrm{a}$ & $94,2 \mathrm{a}$ & $62,2 \mathrm{a}$ & $27,2 \mathrm{a}$ & $4,8 \mathrm{a}$ & $0,50 \mathrm{a}$ \\
$\mathbf{B}$ & 31,0 & $90,9 \mathrm{a}$ & $22,1 \mathrm{a}$ & $90,9 \mathrm{a}$ & $62,3 \mathrm{a}$ & $26,7 \mathrm{a}$ & $4,7 \mathrm{a}$ & $0,48 \mathrm{ab}$ \\
$\mathbf{C}$ & 28,5 & $43,3 \mathrm{~b}$ & $22,6 \mathrm{a}$ & $43,3 \mathrm{~b}$ & $62,5 \mathrm{a}$ & $24,8 \mathrm{a}$ & $4.6 \mathrm{a}$ & $0,47 \mathrm{ab}$ \\
$\mathbf{D}$ & 19,9 & $7,5 \mathrm{c}$ & $23,1 \mathrm{a}$ & $7,5 \mathrm{c}$ & $67,3 \mathrm{~b}$ & $22,3 \mathrm{a}$ & $4,1 \mathrm{~b}$ & $0,39 \mathrm{~b}$ \\
\hline $\mathbf{C V ( \% )}$ & - & $\mathbf{9 , 6 7}$ & $\mathbf{4 , 5 1}$ & $\mathbf{9 , 6 7}$ & $\mathbf{3 , 4 0}$ & $\mathbf{2 2 , 8 3}$ & $\mathbf{7 , 1 6}$ & $\mathbf{1 5 , 4 7}$ \\
\hline
\end{tabular}

*Médias seguidas de mesmas letras, nas colunas, não diferem entre si, pelo teste de Tukey, ao nível de 5\% de probabilidade.

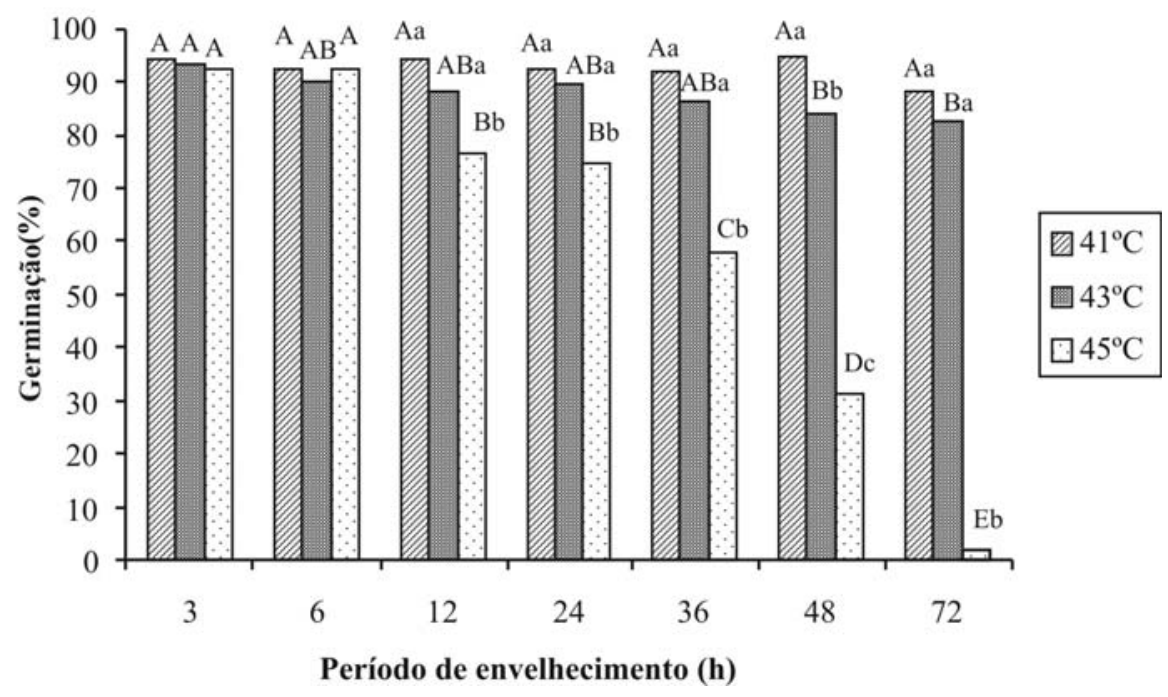

FIGURA 1- Porcentagem de germinação de diásporos de Oenocarpus bacaba Mart. após envelhecimento acelerado a $41 ; 43$ e $45^{\circ} \mathrm{C}$ e cerca de $100 \%$ de umidade relativa do ar, durante $3 ; 6 ; 12 ; 24$; $36 ; 48$ e $72 \mathrm{~h}$.

*Letras maiúsculas para comparação de temperatura entre períodos e minúsculas para comparar temperaturas dentro de cada período, pelo teste de Tukey, ao nível de $1 \%$ de probabilidade. 


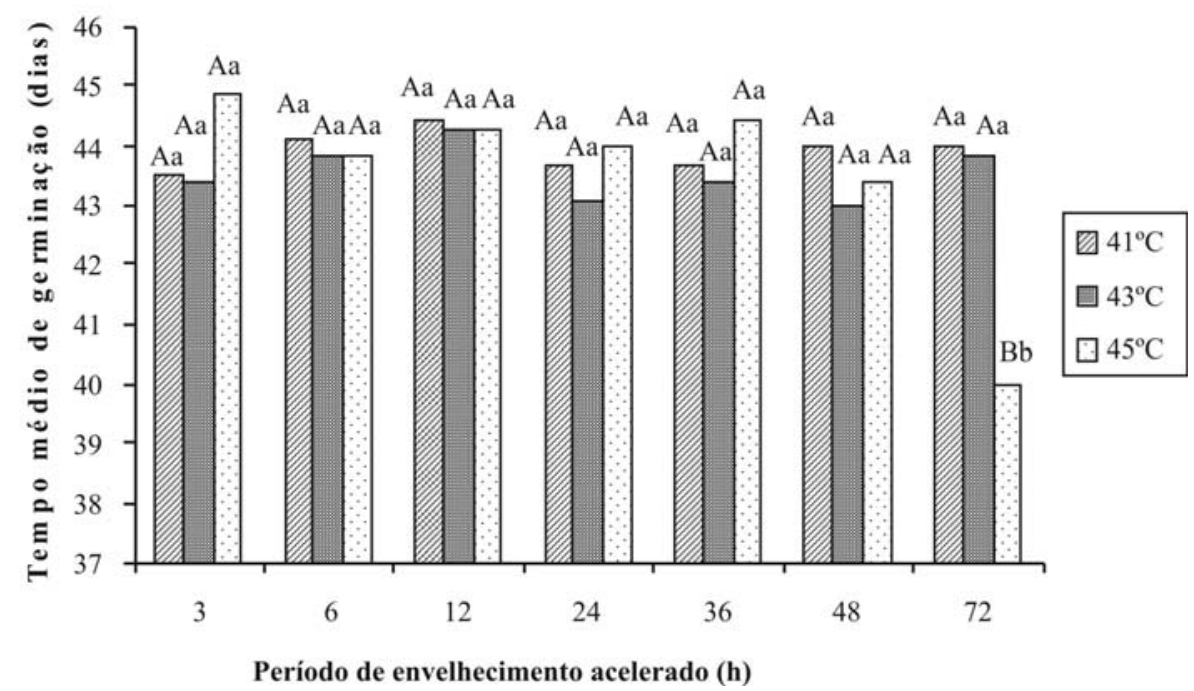

FIGURA 2 -Tempo médio de germinação, em dias, de diásporos de Oenocarpus bacaba Mart. após envelhecimento acelerado de $3 ; 6 ; 12 ; 24 ; 36 ; 48$ e $72 \mathrm{~h}$, a $41 ; 43$ e $45^{\circ} \mathrm{C}$ e cerca de $100 \%$ de umidade relativa do ar, independentemente do critério de avaliação da germinação. Letras maiúsculas para comparação de temperaturas entre períodos e minúsculas entre temperaturas dentro de cada período, pelo teste de Tukey, ao nível de $1 \%$ de probabilidade.

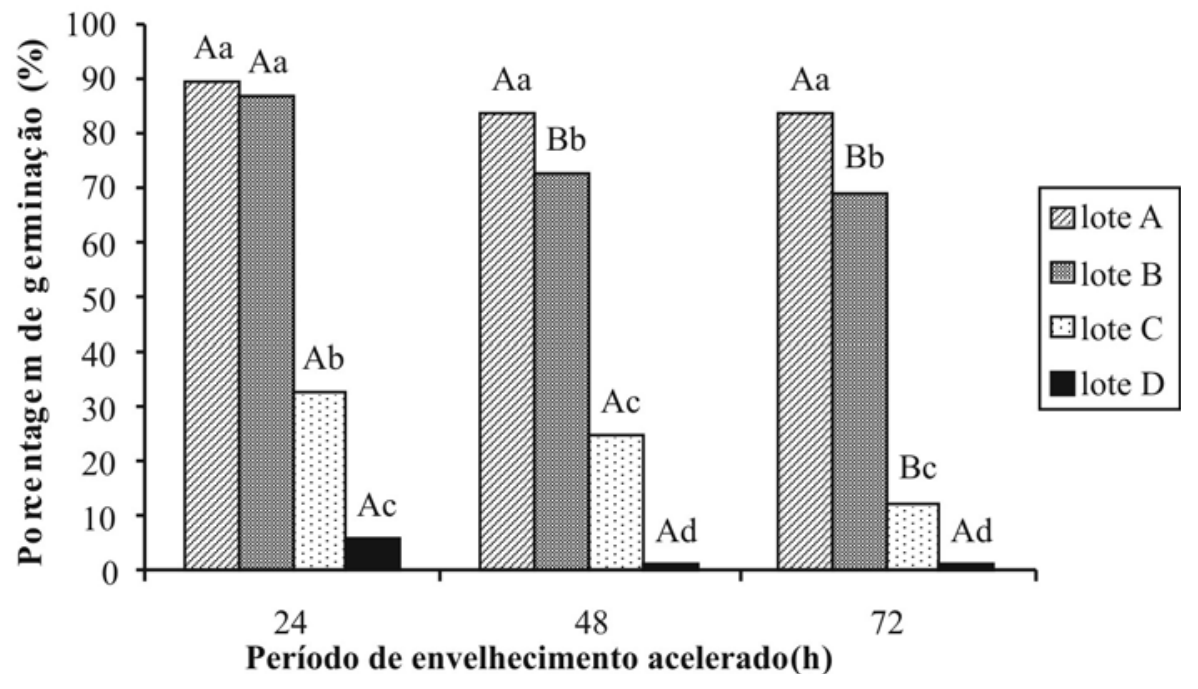

FIGURA 3- Porcentagem de germinação, avaliada pelo critério de emissão da primeira bainha, de diásporos dos lotes de Oenocarpus bacaba Mart. após o envelhecimento acelerado a $43^{\circ} \mathrm{C}$ por 24 ; 48 e $72 \mathrm{~h}$.

* Letras maiúsculas,para comparação de lote entre períodos e letras minúsculas para lotes dentro de cada período, pelo teste de Tukey, ao nível de $1 \%$ de probabilidade. Coeficiente de variação $(\%)=11,92$. 


\section{CONCLUSÃO}

O desenvolvimento da primeira bainha igual ou superior a um centímetro é adequado para avaliar a germinação de diásporos de bacaba após o envelhecimento acelerado. Os lotes de diásporos de bacaba podem ser separados em níveis de vigor por $48 \mathrm{~h}$ de envelhecimento acelerado a $43^{\circ} \mathrm{C}$ e com cerca de $100 \%$ de umidade relativa do ar.

\section{REFERÊNCIAS}

BENINCASA, M.M.P. Análise de crescimento de plantas: noções básicas. 2.ed. Jaboticabal: FUNEP, 2003. 41p.

BRASIL. Ministério da Agricultura e Reforma Agrária. Regras para análise de sementes. Brasília: SNDA/DNPV/CLAV, 1992. 365p.

CARVALHO, N.M.; NAKAGAWA, J. Sementes: ciência, tecnologia e produção. 4: ed. Jaboticabal: FUNEP, 2000. 588p.

CLEMENT, C.R.; BOVI, M.L.A. Padronização de medidas de crescimento e produção em experimento com pupunheiras para palmito. Acta Amazonica, Manaus, v. 30, n. 3, p. 349-362. 2000.

DELOUCHE, J.C.; BASKIN, C.C. Accelerated aging techniques for predicting the relative storability of seed lots. Seed Science and Technology, Zurich, v.1, n. 2, p. 427-452, 1973.

LABOURIAU, L.G.; AGUDO, M. On the physiology of seed germination in Salvia hispanica L. I. Temperature effects. Anais da Academia Brasileira de Ciências. Rio de Janeiro, v.57, p. 37-56, 1987.

MARCOS-FILHO, J. Teste de envelhecimento acelerado. In: KRZYZANOWSKI, F.C.; VIEIRA, R.D.; FRANÇA NETO, J.B. (Eds.). Vigor de sementes: conceitos e testes. Londrina: ABRATES, 1999. p.3.1-3.24.

MARCOS-FILHO, J. Fisiologia de sementes de plantas cultivadas. Piracicaba: FEALQ, 2005. $495 p$.
MARTINS, C.C.; NAKAGAWA, J.; BOVI, M.L.A. Tolerância à dessecação de sementes de palmitovermelho (Euterpe espiritosantensis Fernandes). Revista Brasileira de Botânica, São Paulo, v. 22, n.3, p. 391-396, 1999.

MURUGESAN, P.; MATHUR, R.K.; PILLAI, R.S.N.; KOCHU-BABU, M. Effect of accelerated aging on seed germination of oil palm (Elaeis guineensis Jacq. dura Becc.). Seed Technology, Kentucky, v. 27, n. 1, p. 109-112, 2005.

NEGREIROS, G.F.; PEREZ, S.C.J.G. Resposta fisiológica de sementes de palmeiras ao envelhecimento acelerado. Pesquisa Agropecuária Brasileira, Brasília, v.39, n.4, p.391-396, 2004.

PIMENTEL-GOMES, F. Curso de estatística experimental. Rio de Janeiro: Nobel, 1987. 467p.

SILVA, B.M.S.; CESARINO, F ; LIMA, J.D. ; PANTOJA, T.F. Efeito do substrato e da temperatura na germinação de sementes da Oenocarpus bacaba Mart. (ARECACEAE). In: CONGRESSO BRASILEIRO DE FISIOLOGIA VEGETAL, 10., E CONGRESSO LATINO-AMERICANO DE FISIOLOGIA VEGETAL, 12., 2005, Recife. Anais...

SILVA, B.M.S. Morfo-anatomia e envelhecimento acelerado em diásporos de Oenocarpus bacaba Mart. - ARECACEAE. 2007. 86 f. Dissertação (Mestrado em Agronomia, Produção e Tecnologia de Sementes] - Faculdade de Ciências Agrárias e Veterinárias, Universidade Estadual Paulista, Jaboticabal, 2007.

TEKRONY, D.M. Accelerated aging test. Journal of Seed Technology, Bolise, v.17, p.110-120, 1993.

TOMES, L.J.; TEKRONY, D.M.; EGLI, D.B. Factors influencing the tray accelerated aging test for soybean seed. Journal of Seed Technology, Boise, v. 12, n. 1, p. 24-35, 1988.

TORRES, S.B. Teste de envelhecimento acelerado em sementes de erva-doce. Revista Brasileira de Sementes, Pelotas, v. 26, n. 2, p.20-24, 2004.

VIEIRA, R.D.; CARVALHO, N.M. Testes de vigor em sementes. Jaboticabal: FUNEP, 1994. 164p. 